ARTICLE

\title{
A general approach to engineer positive-going eFRET voltage indicators
}

\author{
Ahmed S. Abdelfattah (10 ${ }^{1 凶}$, Rosario Valenti ${ }^{1}$, Jihong Zheng (1) ${ }^{1}$, Allan Wong ${ }^{1}$, GENIE Project Team*,
} Kaspar Podgorski (D) ${ }^{1}$, Minoru Koyama (1) ${ }^{1}$, Douglas S. Kim ${ }^{1} \&$ Eric R. Schreiter (D) ${ }^{1 \times}$

Imaging membrane voltage from genetically defined cells offers the unique ability to report spatial and temporal dynamics of electrical signaling at cellular and circuit levels. Here, we present a general approach to engineer electrochromic fluorescence resonance energy transfer (eFRET) genetically encoded voltage indicators (GEVIs) with positive-going fluorescence response to membrane depolarization through rational manipulation of the native proton transport pathway in microbial rhodopsins. We transform the state-of-the-art eFRET GEVI Voltron into Positron, with kinetics and sensitivity equivalent to Voltron but flipped fluorescence signal polarity. We further apply this general approach to GEVIs containing different voltage sensitive rhodopsin domains and various fluorescent dye and fluorescent protein reporters.

\footnotetext{
${ }^{1}$ Janelia Research Campus, Howard Hughes Medical Institute, Ashburn, VA, USA. *A full list of consortium members appears at the end of the paper.

凶email: abdelfattaha@janelia.hhmi.org; schreitere@janelia.hhmi.org
} 
G enetically encoded voltage indicators (GEVIs) allow visualization of fast action potentials and subthreshold dynamics in groups of genetically defined neurons with high spatiotemporal resolution ${ }^{1,2}$. Monitoring voltage signals in vivo in large populations of neurons will enable dissection of detailed mechanistic links between brain activity and animal behavior. Despite recent advances, no current GEVI has ideal properties for routine, robust in vivo imaging. Knowledge of the mechanistic function of GEVIs will lead to improved designs. Existing GEVIs use voltage-sensitive protein domains from voltage-sensitive ion channels or phosphatases ${ }^{3-7}$ (VSD GEVIs), or microbial rhodopsin domains $s^{8-14}$ (rhodopsin GEVIs). Archaerhodopsin 3 (Arch) from Halorubrum sodomense was the first rhodopsin GEVI that accurately tracked changes in neuronal membrane potential ${ }^{8}$. However, Arch also generated a hyperpolarizing light-driven current upon exposure to imaging light ${ }^{8,15}$ by functioning as an outward proton pump ${ }^{15}$. A single amino acid substitution (D95N) in Arch abolished light-driven currents and retained Arch voltage-dependent fluorescence change ${ }^{8}$. The equivalent mutation in Ace $1^{12}$, Ace ${ }^{12,14}$, and $\mathrm{Mac}^{13}$ rhodopsins was used to generate later GEVIs.

The rhodopsin GEVI optical signal is fast and linear ${ }^{8}$, two desirable features for a voltage indicator. However, rhodopsin fluorescence is very dim, requiring intense illumination for imaging ${ }^{8}$. Fusions of fluorescent protein (FP) domains or other bright fluorophores to rhodopsin GEVIs were therefore made to facilitate imaging ${ }^{11,13,14}$. These fusions enable voltage-sensitive electrochromic fluorescence resonance energy transfer (eFRET) from a bright fluorophore to the retinal cofactor within the rhodopsin, which acts as a dark quencher. Although the absorbance of the retinal cofactor increases with increasing membrane potential, the emission of the eFRET-coupled fluorophore consequently decreases. All reported eFRET GEVIs therefore have negatively sloped fluorescence-voltage relationships; they are brighter at resting membrane potential and become dimmer during an action potential (negative going). Although two VSD GEVIs, FlicR ${ }^{4}$ and Marina ${ }^{16}$, exhibit positive-going signals in neurons, they have significantly slower response kinetics than eFRET GEVIs, making detection of action potentials difficult. Here we present a general approach to engineer eFRET GEVIs with fast, bright, and positive-going fluorescence signals in response to neuronal action potentials by modification of the natural proton transport pathway within microbial rhodopsins.

\section{Results}

Engineering a positive-going eFRET GEVI. Previous work fused the Ace 2 rhodopsin from Acetabularia acetabulum ${ }^{17}$ to the FP mNeonGreen to produce a negative-going eFRET GEVI that allowed in vivo imaging of voltage signals in several model organisms ${ }^{12}$. We recently used the same Ace2 rhodopsin to engineer a negative-going chemigenetic eFRET GEVI called Voltron, which uses a HaloTag protein domain to covalently bind bright and photostable small-molecule flurophores ${ }^{18,19}$, extending the duration and number of neurons imaged simultaneously in vivo ${ }^{14}$. In both of these GEVIs, photocurrent of Ace2 rhodopsin (Fig. 1a) is blocked by mutating the residue that normally functions as the proton acceptor (PA) ${ }^{20}$ (D81N) (Fig. 1a, b), analogous to the Arch D95N mutation described above. This mutation blocks the primary pathway for exchange of protons from the retinal Schiff base, which links retinal to the rhodopsin protein, to outside the cell ${ }^{20}$. Electrophysiology measurements showing transient inward photocurrents with Ace2 D81N (Fig. 1c) and other rhodopsin-based GEVIs ${ }^{21}$, combined with previous mutagenesis and biochemical data ${ }^{17,20}$, suggest that voltage sensitivity in Ace2 D81N and other eFRET GEVIs results from membrane potential changes altering the equilibrium of protonation between the retinal Schiff base, the proton donor (PD) residue ${ }^{20}$, and the cell cytoplasm (Fig. 1a, b).

We hypothesized that we could alter the local electrochemical potential of protons on the retinal Schiff base and instead establish a protonation equilibrium with the outside of the cell, which would cause the rhodopsin absorbance and eFRET fluorescence to exhibit the opposite response to membrane potential change. Blocking access of protons to the retinal Schiff base from the cell cytoplasm should enable preferential exchange of protons with the outside of the cell. To block access of protons from the cytoplasmic side, we substituted the amino acid at the PD position of Voltron for a neutral residue (D92N). As expected, this substitution led to a block of the transient inward photocurrent of Voltron (Fig. 1d). Importantly, Voltron D92N (as well as other substitutions to neutral residues at the PD position (Supplementary Fig. 1)) showed a positive-going fluorescence signal with membrane depolarization, but with slow kinetics that made it incapable of following neuronal action potentials (Fig. 1d).

We reasoned that the proton pathway between the retinal Schiff base of Voltron D92N and the exterior of the cell was inefficient, resulting in the observed slow kinetics. To improve the efficiency of proton movement towards the outside of the cell, we substituted the amino acid at the PA position for a negatively charged aspartate (N81D), as was present in the original Ace2 rhodopsin sequence. This resulted in an indicator (Voltron N81D D92N) that had sufficient response speed to track action potentials in neurons (Fig. 1e). Critically, Voltron N81D D92N exhibited no steady-state photocurrent (Fig. 1e), showing that it can function as a GEVI without pumping protons across the membrane. Voltron N81D D92N had a transient outward photocurrent (Fig. 1e), confirming that the Schiff base proton was now in equilibrium with the outside of the cell. Although Voltron N81D D92N was suitable for monitoring neuronal action potentials with positive-going fluorescence changes, it showed only $\sim 40 \%$ of the fluorescence change of the negative-going Voltron (Figs. 1e and 2a).

To improve the sensitivity of Voltron N81D D92N we focused on the rest of the proton transport pathway of the Ace2 rhodopsin. We reasoned that proton release (PR) residues ${ }^{20}$ near the outside of the cell should play a critical role in proton movement towards the cell exterior and possibly modulate the protonation equilibrium of the retinal Schiff base. Saturation mutagenesis of the two PR sites (positions 189 and 199 in Ace2) led to a variant with the substitution E199V that had $\sim 2$-fold improved dynamic range over Voltron N81D D92N (Fig. 1f, Fig. 2a, and Supplementary Fig. 2). We decided to name Voltron N81D D92N E199V as Positron due to its positive-going response to membrane depolarization. Compared with Voltron, Positron bound to the fluorescent dye $\mathrm{JF}_{525}$ had similar fluorescence response to voltage steps, but with a positive fluorescence-voltage slope (Fig. 2a and Supplementary Fig. 3). With sub-millisecond on and off time constants (Supplementary Fig. 4 and Supplementary Table 1), Positron clearly reported action potentials in neurons (Fig. 1f) with sensitivity equivalent to that of Voltron. Membrane trafficking of Positron was indistinguishable from Voltron (Supplementary Figs. 5 and 6), and expression of Positron did not alter the electrophysiological parameters of neurons in culture (Supplementary Fig. 7). We observed that Positron was $41 \%$ dimmer than Voltron at resting membrane potential in neurons (Fig. 2b) and bleached at approximately half the rate of Voltron (Fig. 2c), consistent with the hypothesis that at rest, the retinal Schiff base of Positron is more protonated and has a higher absorption, resulting in more eFRET and less dye 
a
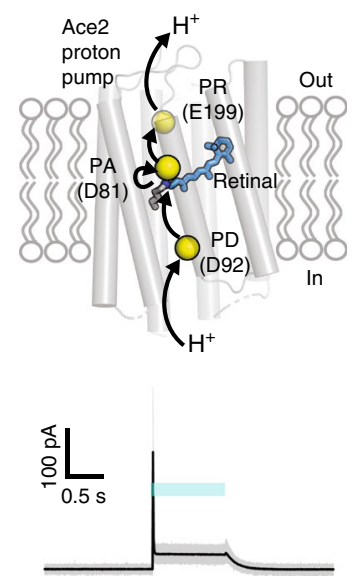

C
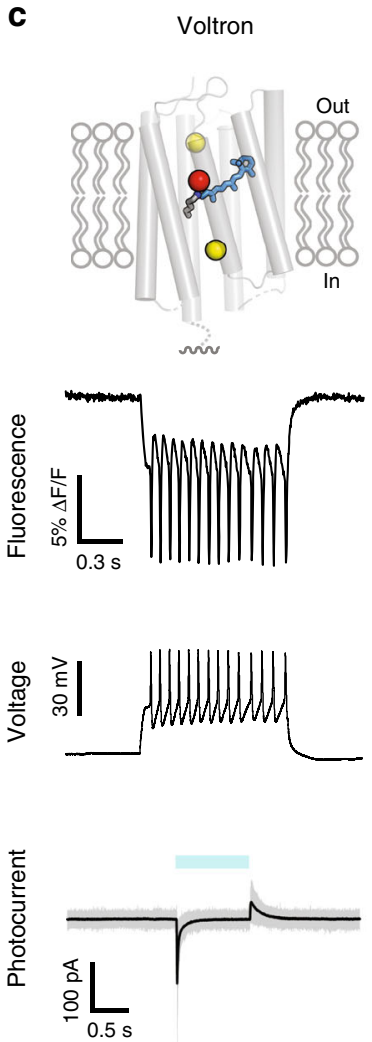

b
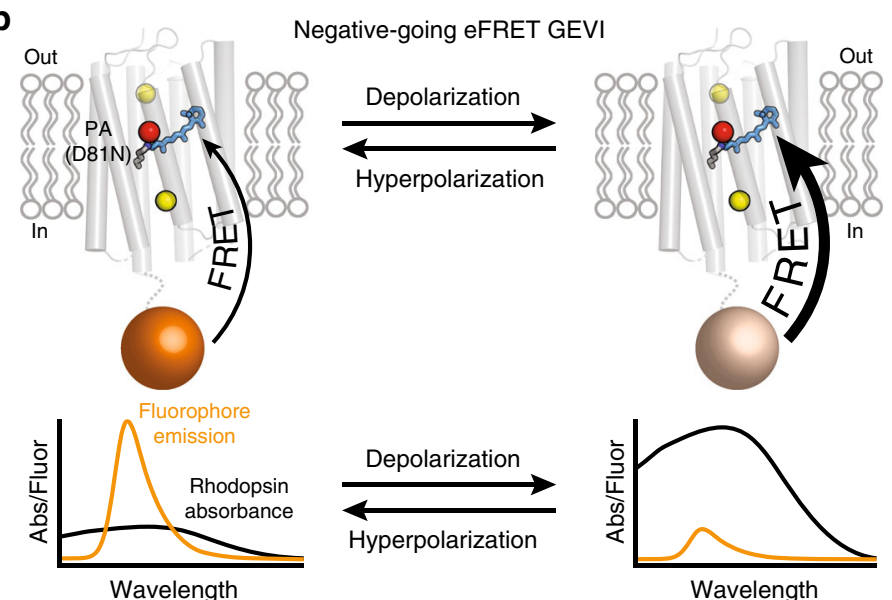

Wavelength

f Voltron N81D D92N E199V (Positron)

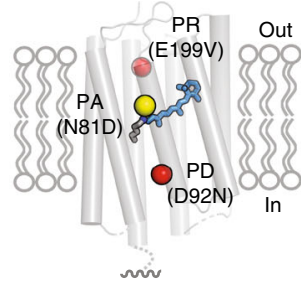

$\min$
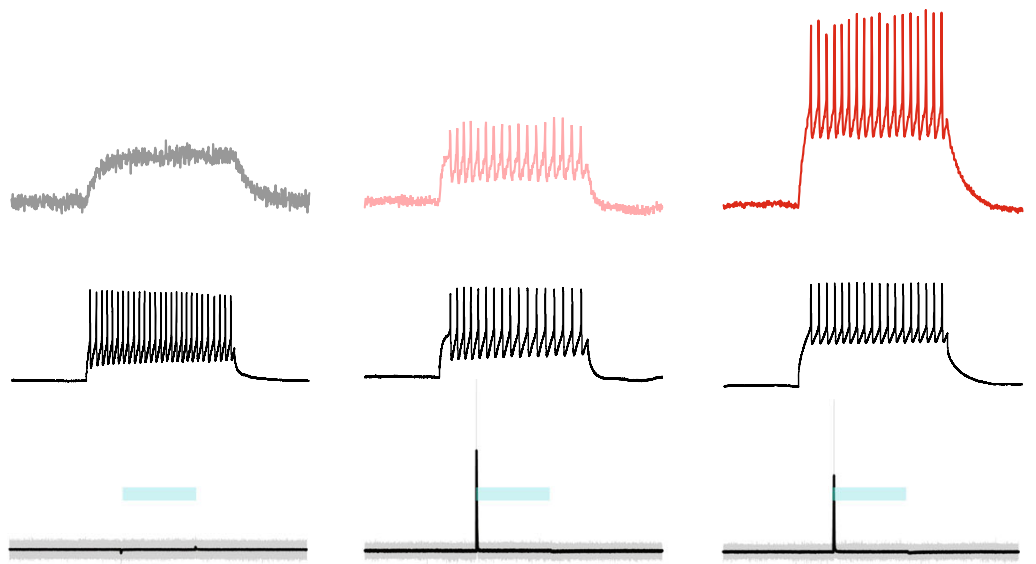

Fig. 1 Engineering a positive-going rhodopsin eFRET GEVI. a Schematic (top) showing the hypothetical path of proton transport (arrows) through the Ace 2 rhodopsin proton pump. The proton acceptor (PA), proton donor (PD), and proton release (PR) positions are represented as yellow spheres and labeled, retinal is shown as blue sticks. Photocurrent measurements (bottom) for the Ace 2 rhodopsin proton pump. Steady-state photocurrent $=46 \pm 11 \mathrm{pA}$ (mean $\pm S D, N=4$ cells). b Schematic representing the proposed mechanism of negative-going rhodopsin eFRET GEVIs. Spectra ${ }^{14}$ in the lower panels have been scaled to illustrate the direction of absorbance and fluorescence change with membrane voltage change. We were not able to quantitatively measure these spectra in cell membranes at different voltages. At resting membrane potential, rhodopsin absorbance is low and therefore fluorophore emission is high. When the membrane depolarizes, rhodopsin absorbance increases leading to a decrease in fluorophore emission. $\mathbf{c}-\mathbf{f}$ Schematic of amino acid substitutions (top), simultaneous fluorescence imaging (second row), and whole-cell patch-clamp membrane voltage measurements (third row), as well as photocurrent measurements (bottom) from rat hippocampal neurons in culture expressing Voltron, Voltron D92N, Voltron D92N N81D, and Positron labeled with $\mathrm{JF}_{525}$. Blue bar denotes time of light illumination $\left((508 \mathrm{~nm}-522 \mathrm{~nm})\right.$ at an irradiance of $70 \mathrm{~mW}$ mm $\left.{ }^{-2}\right)$ for photocurrent measurements. Steady-state photocurrents for all variants are negligible $(-0.7 \pm 3 p A,-0.1 \pm 1 p A, 0.0 \pm 1.5 p A, 0.1 \pm 1 p A$ (mean $\pm S D, N=5-7$ cells), respectively). Simultaneous voltage and fluorescence traces are representative of $N \geq 3$ cells.

fluorescence emission. When the membrane depolarizes, the proton is driven toward the outside of the cell, resulting in lower retinal absorption, less quenching of dye fluorescence, and a positive-going fluorescence response (Fig. 2d).
Using Positron for in vivo voltage imaging. We previously showed that Voltron was suitable for imaging voltage signals in the brains of live animals such as fruit flies, zebrafish, and mice ${ }^{14}$. To confirm that Positron allows for in vivo imaging, we recorded 

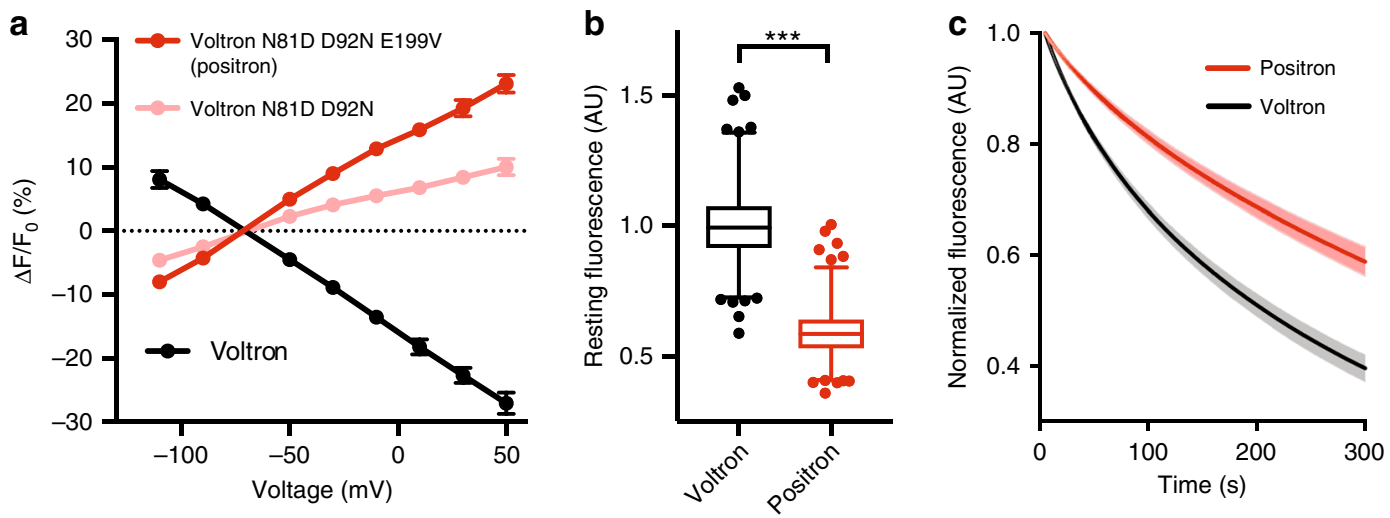

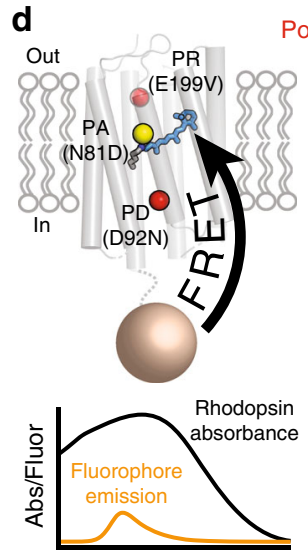

Wavelength
Positive-going eFRET GEVI

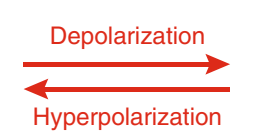$$
\text { 列 }
$$
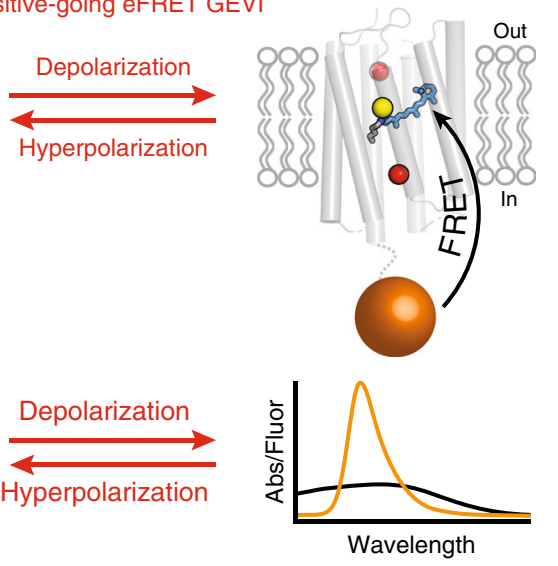

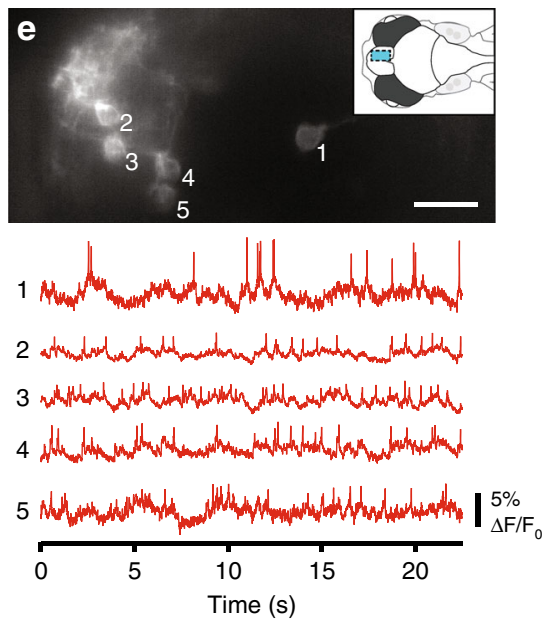

Fig. 2 Characterization of positron neuron culture and larval zebrafish. a Graph of fluorescence vs. membrane voltage for $V$ oltron ( $N=6$ cells), $V$ oltron D92N N81D ( $N=5$ cells), and Positron ( $N=5$ cells) expressed in voltage-clamped rat hippocampal neurons in culture. $\Delta F / F_{0}$ measurements are mean \pm

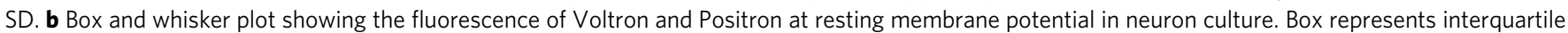

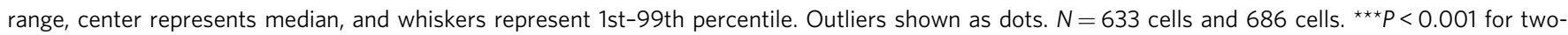
tailed unpaired t-test. c Comparison of photobleaching of Positron and Voltron. Mean normalized fluorescence from a field of view of labeled, GEVIexpressing neurons is shown \pm SEM. $N=19$ fields of view each from $N=4$ independent neuron cultures each. d Schematic representing the proposed mechanism of positive-going rhodopsin eFRET GEVIs. Spectra ${ }^{14}$ in the lower panels have been scaled to illustrate the direction of absorbance and

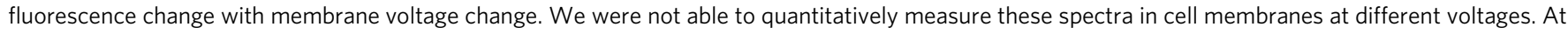

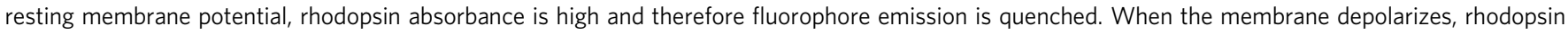
absorbance decreases leading to an increase in fluorophore emission. e Imaging of voltage signals from five neurons in the forebrain of live larval zebrafish.

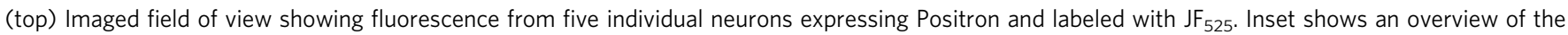
larval zebrafish brain and the imaged area (blue rectangle). (bottom) Fluorescence of the five visible neurons over time. Scale bar: $20 \mu \mathrm{m}$ Data are representative of $N=3$ fish.

optical voltage signals from five neurons simultaneously in the forebrain of a larval zebrafish expressing Positron and labeled with $\mathrm{JF}_{525}$ using a wide-field fluorescence microscope (Fig. 2e). We observed independent spiking and subthreshold signals in each of the neurons (Fig. 2e) with signals similar to those observed with Voltron previously in the same preparation (compare Fig. 2e with Fig. S34 of ref. ${ }^{14}$ ). We also directly compared Positron and Voltron imaging of spontaneous action potential spikes in olfactory sensory neurons (OSNs) of larval zebrafish (Supplementary Fig. 8). Positron and Voltron provided equivalent SNR in this preparation and each showed robust signals at the end of the five-minute imaging window, suggesting that imaging could likely continue for significantly longer. We have only performed one-photon in vivo imaging, since Voltron and Positron, such as other rhodopsin-based GEVIs, are not compatible with two-photon imaging 7,14 . In neuron cultures, fluorescence responses to action potentials decayed at a similar rate for Positron and Voltron (Supplementary Fig. 9). Although currently Positron and Voltron perform interchangeably in vivo, we ran simulations to assess how future imaging of positive-going or negative-going GEVIs would be influenced by the density of labeled neurons and the sensitivity of the indicator, which our current experiments could not address. We found that for more densely labeled samples with higher background and for higher sensitivity indicators, positive-going GEVIs significantly outperform equivalent negative-going indicators (Supplementary Fig. 10). For example, GEVIs with a $5 \times$ larger fluorescence response to a neuron action potential allow imaging in $4 \times$ more densely labeled tissue using a positive-going indicator relative to a negative-going indicator with the same spike detection error rate (Supplementary Fig. 10c, d). Moreover, at high labeling density, the more sensitive positive-going indicator produces a $4 \times$ higher spike detection fidelity than its negative-going counterpart in our simulations (Supplementary Fig. 10f).

A general engineering method for positive-going eFRET GEVIs. To demonstrate the generality of our approach to 
a
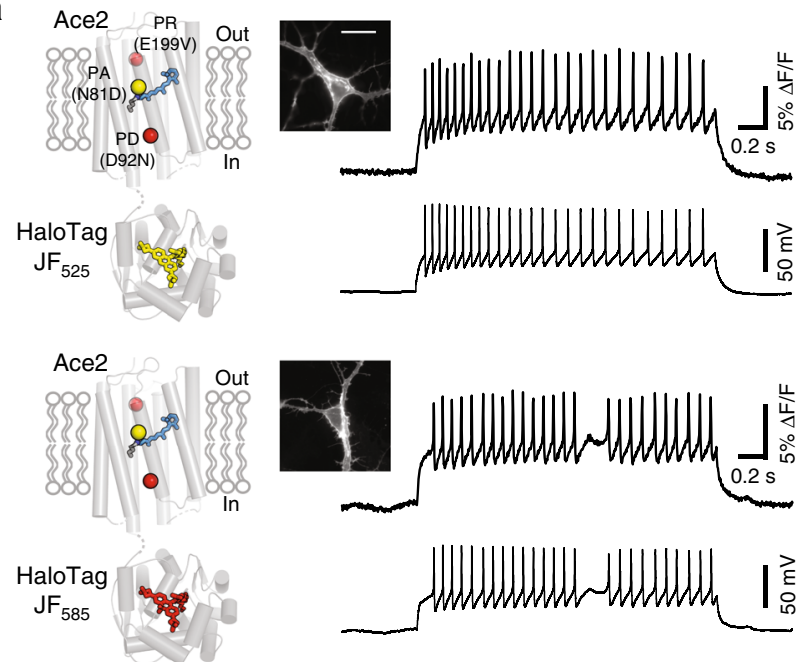

b
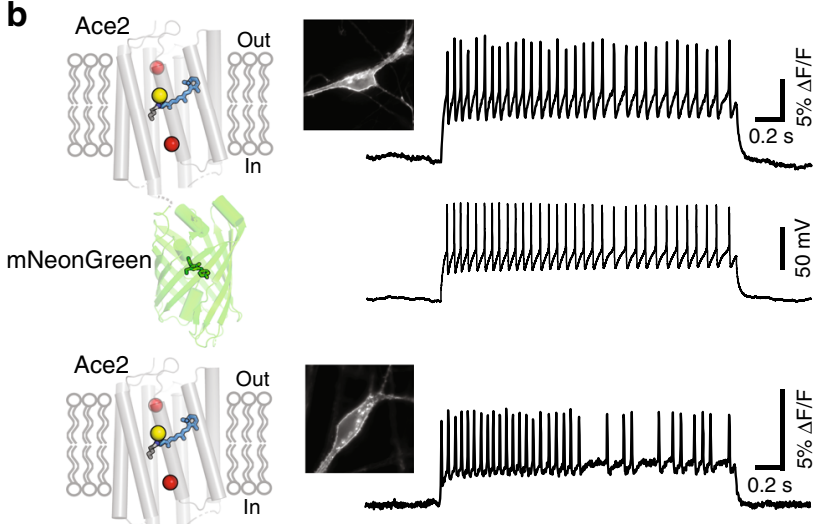

mRuby3
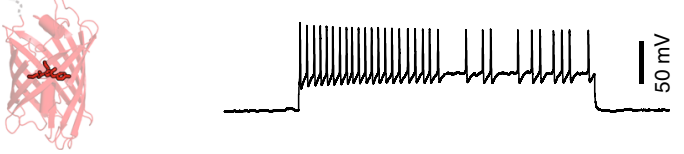

C
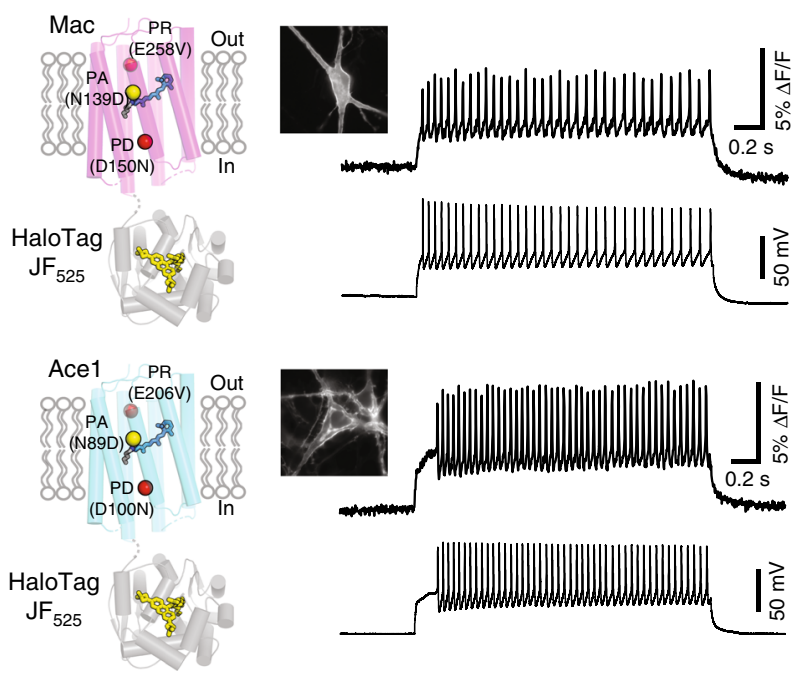

Fig. 3 Generality of the approach to engineer positive-going eFRET GEVIs. Each panel shows a schematic of the voltage indicator construct (left), a fluorescence image of a neuron in culture expressing this voltage indicator (center), and simultaneous recording of fluorescence (right top) and membrane potential (right bottom) in response to current injection. Fluorescence image scale bar $=20 \mu \mathrm{m}$. Simultaneous voltage and fluorescence traces are representative of $N \geq 3$ cells. a Positron labeled with two different colors of fluorescent dye, $\mathrm{JF}_{525}$ (top) and $\mathrm{JF}_{585}$ (bottom). b Ace 2 rhodopsin bearing the signal-inverting mutations described and fused to the different color FP domains mNeonGreen (top) and mRuby3 (bottom). c Different rhodopsin domains (Mac, top and Ace1, bottom) bearing mutations analogous to the described signal-inverting mutations described, fused to HaloTag, and labeled with $\mathrm{JF}_{525}$.

of the Ace2N-mNeon ${ }^{12}$ and VARNAM ${ }^{22}$ eFRET GEVIs capable of sensitively reporting action potentials in neurons with positivegoing signals (Fig. $3 \mathrm{~b}$ and Supplementary Figs. 11 and 12). Next, we substituted the rhodopsin domain with Acel, Mac, and Arch rhodopsins bearing mutations analogous to the three mutations we introduced to create Positron (Fig. 3c and Supplementary Figs. 13-18). Despite only low amino acid sequence identity (27-54\%) between these rhodopsins (Supplementary Fig. 16), each of these permutations resulted in a GEVI with positive-going fluorescence changes capable of following action potentials when we patched neurons in culture and injected current (Fig. $3 \mathrm{c}$ and Supplementary Fig. 18). Importantly, all three rhodopsins bearing mutations analogous to Positron showed no steady-state photocurrent (Supplementary Figs. 17 and 18).

\section{Discussion}

Positron is the first eFRET GEVI to run in reverse, having lower fluorescence at resting membrane potentials, positive-going signals in response to action potentials, and sensitivity equal to that of state-of-the-art eFRET GEVIs. We achieved this by rational mutation of the PD, PA, and PR sites within the characterized proton transport pathway of microbial rhodopsins and found that the effect of these substitutions can be generalized to other rhodopsins. This work provides further mechanistic insight into the class of rhodopsin eFRET GEVIs and puts forth a scaffold for future GEVI optimization.

\section{Methods}

Molecular biology. The genes for Ace2 and HaloTag were amplified from a Voltron plasmid ${ }^{14}$. The genes for Ace1, MacQ, QuasAr3, mNeonGreen, and mRuby3 were synthesized (Integrated DNA Technologies) with mammalian codon optimization $^{10-13,22}$. Rhodopsin and fluorescence reporter genes were combined using overlap PCR. Cloning was done by restriction enzyme digest of plasmid backbones, PCR amplification of inserted genes, isothermal assembly, and followed by Sanger sequencing to verify DNA sequences. A soma localization $\operatorname{tag}^{23,24}$ (ST) was added using overlap PCR to make soma targeted versions of indicators (e.g. Positron-ST). For expression in primary neuron cultures, sensors were cloned into a pcDNA3.1-CAG plasmid (Invitrogen). For expression in zebrafish, Positron-ST was cloned into the pTol2-HuC vector (for pan-neuronal expression) and into the pT2-Tbait-UAS vector (for Gal4-dependent expression). The DNA and amino acid sequences of Positron, Ace1_Q89D_D100N_E206V-HaloTag, QuasAr3_Q95D_H106N_E214V-HaloTag, Mac_Q139D_D150N_E258V-HaloTag, Ace2_D92N_E199V-mNeonGreen, Ace2_D92N_E199V-mRuby3, are given in Supplementary Figs. 2 and 11-15. Plasmids and maps are available from Addgene.

HEK 293 cell culture. Spiking HEK 293 cells $^{25}$ that stably express NaV1.3 and Kir2.1 were used to test the fluorescence response of some Positron mutants using field stimulation. Cells were grown at $37^{\circ} \mathrm{C}, 5 \% \mathrm{CO}_{2}$, in Dulbecco's modified Eagle medium supplemented with 10\% fetal bovine serum, Geneticin (Invitrogen, 10131027), Penicillin/Streptomycin (Invitrogen, 15140-122), and Puromycin (Invitrogen, A11138-03). All the transfections and assays were done in cells between passage 5 and 15. Plasmids were transfected using calcium phosphate. After transfection, spiking HEK cells were plated on glass-bottom 24-well plates (MaTeK) and generate eFRET GEVIs with positive-going fluorescence response, we explored different reporter fluorophores and rhodopsin domains. Positron showed sensitive fluorescence response when labeled with a yellow dye $\left(\mathrm{JF}_{525}\right)$ or a red dye $\left(\mathrm{JF}_{585}\right)$ (Fig. 3a). We also showed that the HaloTag could be exchanged for either green or red FP domains (Fig. 3b). We created positive-going versions 
cultured for $24 \mathrm{~h}$ before imaging. To label HaloTag-containing constructs, cells were incubated with $100 \mathrm{nM}$ JF-dye HaloTag ligand for $30 \mathrm{~min}$.

Primary neuron cell culture. All procedures involving animals were conducted in accordance with protocols approved by the Howard Hughes Medical Institute Janelia Research Campus Institutional Animal Care and Use Committee and Institutional Biosafety Committee. Hippocampal neurons extracted from P0 to 1 Sprague-Dawley rat pups were transfected with pcDNA3.1-CAG plasmids of the various indicators by electroporation (Lonza, P3 Primary Cell 4D-Nucleofector X kit) according to the manufacturer's instruction. After transfection, hippocampal neurons were plated onto 24-well glass-bottom plates (MaTek) or $35 \mathrm{~mm}$ glassbottom dishes (MaTek) coated with poly-D-lysine (Sigma). Neurons were cultured for 8-12 days in NbActiv4 medium (BrainBits). To label neurons expressing Positron, Voltron, Ace1_Q89D_D100N_E206V-HaloTag, QuasAr3_Q95D_H106N_E214V-HaloTag, and Mac_Q139D_D150N_E258V-HaloTag, cultures were incubated with $100 \mathrm{nM}$ JF HaloTag ligand for 20-30 mins.

Microscopy. Cell cultures were illuminated with a SPECTRA X light engine (Lumencore) and observed through a $\times 40$ oil objective (NA $=1.3$, Nikon) on an inverted Nikon Eclipse Ti2 microscope. Excitation and emission light were passed through a FITC filter set $(475 / 50 \mathrm{~nm}$ (excitation), $540 / 50 \mathrm{~nm}$ (emission), and a 506LP dichroic mirror (FITC-5050A-000; Semrock)) to image Ace2_D92N_E199VmNeonGreen, a custom filter set (510/25 nm (excitation), 545/40 nm (emission), and a 525LP dichroic mirror (Chroma)) to image Voltron, Positron, Acel Q89D D100N_E206V-HaloTag, and Mac_Q139D_D150N_E258V-HaloTag labeled with JF 525 , and a quad bandpass filter (set number: 89000 , Chroma) with the appropriate color band from the SPECTRA X light source to image QuasAr3_Q95D_H106N_E214VHaloTag labeled with $\mathrm{JF}_{549}$, Positron labeled with $\mathrm{JF}_{585}$, and Ace2_D92N_E199VmRuby3. Fluorescence was collected onto a scientific CMOS camera (ORCA-Flash 4.0, Hamamatsu) and acquired using HCImage Live (Hamamatsu). For time-lapse imaging, images were acquired at $400-3200 \mathrm{~Hz}$ depending on the experiment. Imaging buffer (containing the following (in $\mathrm{mM}$ ): $145 \mathrm{NaCl}, 2.5 \mathrm{KCl}, 10$ glucose, 10 HEPES $\mathrm{pH} 7.4,2 \mathrm{CaCl}_{2}, 1 \mathrm{MgCl}_{2}$ ) was used in all cell culture experiments.

To process fluorescence images, a region of interest (ROI) was manually drawn around a neuron cell body in image and the fluorescence was measured by averaging all pixels within the cell body. The raw fluorescence trace $(F)$ is the mean intensity over the ROI in time. $F$ is then fit with an exponential curve to account for bleaching. We calculated $\Delta F / F$ as $F-F_{0} / F_{0}$ with $F_{0}$ being the fluorescence baseline averaged over one second prior to stimulation.

To compare brightness of Positron and Voltron at resting membrane potential, we fused mTagBFP2 ${ }^{26}$ to the $\mathrm{C}$ terminus of Positron and Voltron, to make pCAGPositron-mTagBFP2 and pCAG-Voltron-mTagBFP2 plasmids. Fluorescence images were acquired from ten different wells across three independent transfections for each construct in primary hippocampal neurons. To label Voltron-mTagBFP2- and Positron-mTagBFP2-expressing neurons, cultures were incubated with $100 \mathrm{nM} \mathrm{JF}_{525}$ HaloTag ligand for $25 \mathrm{~min}$, then washed twice with imaging buffer, incubating the cells for 5 min during the second wash. An EBFP2 filter set (405/20 nm (excitation), 460/50 nm (emission), and a 425LP dichroic mirror (49021; Chroma)) was used to image the TagBFP2 channel, and a custom filter set $(510 / 25 \mathrm{~nm}$ (excitation), 545/40 nm (emission), and a 525LP dichroic mirror (Chroma)) was used to image the $\mathrm{JF}_{525}$ channel in Voltron-mTagBFP2 and Positron-mTagBF2 constructs. The ratio of the TagBFP2 channel and $\mathrm{JF}_{525}$ channel was calculated using ImageJ software.

To compare the photostability of Positron and Voltron at resting membrane potential, we used the soma localized versions, labeled with $\mathrm{JF}_{525}$ HaloTag ligand. Fluorescence images ( $100 \mathrm{~ms}$ exposure) were taken using a $\times 40$ objective every second for $5 \mathrm{~min}$ of neuron cultures exposed to continuous $18 \mathrm{~mW} \mathrm{~mm}^{-2}$ light illumination from a light-emitting diode (LED) (Spectra X, Lumencore). After background subtraction, the average intensity per frame was calculated and plotted vs. time.

Field stimulation in spiking HEK cells or neuron culture. A stimulus isolator (A385, World Precision Instruments) with platinum wires was used to deliver field stimuli $(50 \mathrm{~V}, 1 \mathrm{~ms})$ to elicit HEK cell spiking or action potentials in cultured neurons ${ }^{27}$. The stimulation was controlled using Wavesurfer and timing was synchronized with fluorescence acquisition using Wavesurfer and a National Instruments PCIe-6353 board.

To measure change of Positron and Voltron fluorescence response to action potentials over time, we labeled neurons expressing these indicators and labeled with $\mathrm{JF}_{525}$ HaloTag ligand and imaged with a $\times 40$ objective at $400 \mathrm{~Hz}$ at $60 \mathrm{~mW}$ $\mathrm{mm}^{-2}$. We applied field electrode stimulations to induce a single action potential every $3 \mathrm{~s}$. Individual neurons were manually segmented by drawing ROIs around the cell body. Change in fluorescence was calculated for each action potential of the trace as described above.

Electrophysiology in primary neuron culture. Simultaneous whole-cell patchclamp recordings along with fluorescence imaging were performed in cultured neurons. Current-clamp recordings were performed in imaging buffer: $145 \mathrm{NaCl}$, $2.5 \mathrm{KCl}, 10$ glucose, $10 \mathrm{HEPES}$ pH 7.4, $2 \mathrm{CaCl}_{2}, 1 \mathrm{MgCl}_{2}$, adjusted to $310 \mathrm{mOsm}$ with sucrose at room temperature. For voltage-clamp recordings, $500 \mathrm{nM}$ TTX was added to the imaging buffer to block sodium channels, and synaptic blockers (10 $\mu \mathrm{M}$ CNQX, $10 \mu \mathrm{M}$ CPP, $10 \mu \mathrm{M}$ GABAZINE, and $1 \mathrm{mM} \mathrm{MCPG}$ ) were added to block ionotropic glutamate, GABA, and metabotropic glutamate receptors ${ }^{27}$.

Patch pipettes were fabricated using a P-97 puller (Sutter Instruments) to a tip resistance of 4-6 $\mathrm{M} \Omega$. For current-clamp recordings, pipettes were filled with the following (in $\mathrm{mM}$ ): 130 potassium methanesulfonate, 10 HEPES, $5 \mathrm{NaCl}, 1 \mathrm{MgCl}_{2}$, 1 Mg-ATP, 0.4 Na-GTP, 14 Tris-phosphocreatine, adjusted to $\mathrm{pH} 7.3$ with $\mathrm{KOH}$ and adjusted to $300 \mathrm{mOsm}$ with sucrose. For voltage-clamp recordings, a cesiumbased internal solution was used ((in mM): 115 cesium methanesulfonate, 10 HEPES, $5 \mathrm{NaF}, 10$ EGTA, $15 \mathrm{CsCl}, 3.5 \mathrm{Mg}$-ATP, 3 QX-314, adjusted to $\mathrm{pH} 7.3$ with $\mathrm{CsOH}$, and adjusted to $300 \mathrm{mOsm}$ with sucrose).

Whole-cell recordings were made using an EPC800 amplifier (HEKA), filtered at $10 \mathrm{kHz}$ with the internal Bessel filter, and digitized using a National Instruments PCIe-6353 acquisition board. Pipettes were positioned with a MPC200 manipulator (Sutter Instruments). Wavesurfer software was used to generate current injection waveforms, to record voltage and current traces, and to control the camera and light source. Neurons with access resistance $>25 \mathrm{M} \Omega$ were discarded. For currentclamp recordings to generate action potentials, $20-200 \mathrm{pA}$ for $1-2 \mathrm{~s}$ was injected and voltage was monitored.

For voltage-clamp experiments used to generate fluorescence-voltage curves, voltage steps (from $-110 \mathrm{mV}$ to $+50 \mathrm{mV}$ in $20 \mathrm{mV}$ increments for $1 \mathrm{~s}$ ) were applied to cells held at $-70 \mathrm{mV}$. Fluorescence images were acquired at $400 \mathrm{~Hz}$ using the same microscope described in the "Microscopy" section above. For determining response speed of indicators, fluorescence images were acquired at $3200 \mathrm{~Hz}$ in response to a $100 \mathrm{mV}$ potential step delivered to voltage-clamped neurons (from $-70 \mathrm{mV}$ to $+30 \mathrm{mV}$ ). Traces were fit to a double exponential function using MATLAB. All recordings were done at room temperature.

Photocurrent measurements. Photocurrents were recorded at room temperature in voltage-clamp mode with a holding potential of $-70 \mathrm{mV}$ in response to $1 \mathrm{~s}$ light pulses. Photocurrents were recorded using an EPC800 amplifier (HEKA), filtered at $10 \mathrm{kHz}$ with an internal Bessel filter, and digitized using a National Instruments PCIe-6353 acquisition board at $20 \mathrm{kHz}$ controlled using WaveSurfer. Light was delivered to the clamped neurons using the same microscope described above. Irradiance at the imaging plane was set to $70 \mathrm{~mW} \mathrm{~mm}^{-2}$ determined with a microscope slide power sensor (S170C, Thorlabs).

Imaging in zebrafish. In vivo wide-field voltage imaging in zebrafish was performed. Briefly, the Positron-ST indicator was transiently expressed by the injection of pT2-Tbait-UAS-Positron-ST ( $25 \mathrm{ng} \mathrm{\mu l}^{-1}$ DNA with $25 \mathrm{ng} \mathrm{\mu l}^{-1}$ Tol2 transposase mRNA in E3 medium) in $\mathrm{Tg}$ (elavl3:Gal4-VP16) at 1-2 cell stage. $\mathrm{JF}_{525}$ was loaded to the injected fish at three day post-fertilization (dpf) by incubation in $3 \mu \mathrm{M} \mathrm{JF}_{525}$ system water solution for $2 \mathrm{~h}$. The fish with sparsely labeled forebrain neurons were paralyzed by a-bungarotoxin $\left(1 \mathrm{mg} \mathrm{ml}^{-1}\right)$ and mounted in lowmelting point agarose. Spontaneous activity of forebrain neurons was imaged using a custom wide-field microscope equipped with a $\times 601.0 \mathrm{NA}$ water immersion objective lens (MRD07620, Nikon), a LED light source (CBT-90-W, Luminous) and a TRITC filter set (TRITC-B-000, Semrock). The images were acquired with sCMOS camera (pco.edge 4.2, PCO) at $400 \mathrm{~Hz}$ for 1-2 min. Irradiance at the imaging plane was $28 \mathrm{~mW} \mathrm{~mm}^{-2}$ (S170C, Thorlabs).

To compare the performance of the Positron-ST indicator and the Voltron-ST indicator to detect spiking activity, we focused on OSNs that showed tonic spontaneous spiking activity similar to those reported in other species ${ }^{28,29} . \mathrm{JF}_{552}$ HaloTag ligand ${ }^{30}$ was loaded using the same protocol described above and the fish with sparsely labeled OSNs were selected for imaging in order to minimize the contamination of signals from nearby cells. Using the same imaging protocol described above, fluorescent signal was recorded for $5 \mathrm{~min}$. Raw fluorescent time course of individual OSNs was first extracted from manually drawn ROI and then the bleaching time constant of the dye for each indicator was calculated by fitting an exponential decay function to the raw time course. To examine percent signal change of transient spike-like signal, the baseline fluorescence signal corresponding to the resting membrane potential was estimated by the bottom 10th percentile of a $1 \mathrm{~s}$ moving time window (or the top 10th percentile signal in case of Voltron-ST) and used to calculate percent signal change for spike-like signal $\left(\Delta F / F_{0}\right)$. Then the spike-like events were identified with the following procedure. First, $\Delta F / F_{0}$ was $z$ transformed and then maximal overlap discrete wavelet transform was performed using sym 4 wavelet down to the sixth level. Then a frequency-localized version of the signal was reconstructed using the wavelet coefficients from the level 3 to 6 to maximize the energy of spike-like signal and then a threshold was applied its power to detect spike-like event. This procedure allowed us to detect clear spike-like signal independent of its percent signal change for both indicators with the exact same procedure and threshold. We restricted our analysis to the cells that showed tonic firing throughout the 5-min imaging session and examined amplitude $\left(\Delta F / F_{0}\right)$ and SNR ( $z$-score).

Simulations of positive- and negative- going GEVIs. To simulate performance of positive- vs. negative-going indicators, we started with a real recording from superficial mouse cortex and added simulated neurons as in our previous work ${ }^{14}$. In these simulations, we used a real recording (40,000 frames) containing a single 
Voltron-labeled neuron as a positive control. We synthesized spatial footprints for each neuron. We then simulated 'voltage traces' for each neuron (Supplementary Fig. 10b), containing a mean of 100 spikes per recording, and converted these to either positive and negative-going indicator traces ranging between $F_{\min }$ and $F_{\max }$. For positive-going traces, $F_{\min }$ corresponds to the fluorescence at the resting membrane potential and $F_{\max }$ to the mean spike height. For negative-going traces, $F_{\min }$ corresponds to the mean spike height and $F_{\max }$ to the fluorescence at the resting membrane potential.

Indicator traces were multiplied by the empirical bleaching curve estimated from the neuron in the video and scaled to a comparable brightness. These model neuron recordings were summed across neurons and Gaussian noise proportional to signal was added to each pixel. This sampled simulated recording was then added to the real recording to produce the simulation instance for analysis.

We simulated imaging of different numbers of neurons with different indicator sensitivities. For each simulated neuron for which we estimated spikes, we included 5 additional 'out of focus' neurons with blurred spatial footprints and temporally shuffled spiking intended to model background sources in real recordings. For different indicator sensitivities $\left(\Delta F / F_{\min }\right)$, we held $F_{\max }$ fixed (to simulate a fixed maximum brightness of the dye ligand) and varied $\mathrm{F}_{\min }$ to produce the appropriate $\Delta F / F_{\min }$. Spikes were recovered using the Spike Pursuit algorithm ${ }^{14}$. We plot the error rate of spike inference, calculated as 1-IoU, where IoU is the intersection divided by the union of binary spike labels for the ground truth and inferred spikes at the simulated imaging rate $400 \mathrm{~Hz}$, without binning. Code used in simulations is available from github.com/KasparP/Positron.

Reporting summary. Further information on research design is available in the Nature Research Reporting Summary linked to this article.

\section{Data availability}

All DNA and protein sequences described have been deposited at Addgene (www. addgene.org) as follows: pCAG-Positron (plasmid\# 129253), pCAG-Positron-ST (plasmid\# 129254), pCAG-Ace1_Q89D_D100N_E206V-HaloTag (plasmid\# 129255), pCAG-Ace1_Q89D_D100N_E206V-HaloTag-ST (plasmid\# 129260), pCAGAce2_D92N_E199V-mNeon (plasmid\# 129256), pCAG-Ace2_D92N_E199V-mNeon-ST (plasmid\# 129261), pCAG-Ace2_D92N_E199V-mRuby3 (plasmid\# 129257), pCAGAce2_D92N_E199V-mRuby3-ST (plasmid\# 129262), pCAG-

QuasAr3_Q95D_H106N_E214V-HaloTag (plasmid\# 129258), pCAGQuasAr3_Q95D_H106N_E214V-HaloTag-ST (plasmid\# 129263), pCAGMac_Q139D_D150N_E258V-HaloTag (plasmid\# 129259), pCAGMac_Q139D_D150N_E258V-HaloTag-ST (plasmid\# 129264), pTol2-Huc-Positron-ST (plasmid\# 129265), pT2-Tbait-UAS-Positron-ST (plasmid\# 129266), and pAAV-hsynflex-Positron-ST (plasmid\# 129267). Source data from experiments in this study are available from the authors upon reasonable request.

Received: 8 May 2020; Accepted: 18 June 2020;

Published online: 10 July 2020

\section{References}

1. Xu, Y., Zou, P. \& Cohen, A. E. Voltage imaging with genetically encoded indicators. Curr. Opin. Chem. Biol. 39, 1-10 (2017).

2. Lin, M. Z. \& Schnitzer, M. J. Genetically encoded indicators of neuronal activity. Nat. Neurosci. 19, 1142-1153 (2016).

3. Jin, L. et al. Single action potentials and subthreshold electrical events imaged in neurons with a fluorescent protein voltage probe. Neuron 75, 779-785 (2012).

4. Abdelfattah, A. S. et al. A bright and fast red fluorescent protein voltage indicator that reports neuronal activity in organotypic brain slices. J. Neurosci. 36, 2458-2472 (2016).

5. St-Pierre, F. et al. High-fidelity optical reporting of neuronal electrical activity with an ultrafast fluorescent voltage sensor. Nat. Neurosci. 17, 884-889 (2014).

6. Yang, H. H. H. et al. Subcellular imaging of voltage and calcium signals reveals neural processing in vivo. Cell 166, 245-257 (2016).

7. Chamberland, S. et al. Fast two-photon imaging of subcellular voltage dynamics in neuronal tissue with genetically encoded indicators. Elife 6, e25690 (2017).

8. Kralj, J. M., Douglass, A. D., Hochbaum, D. R., Maclaurin, D. \& Cohen, A. E. Optical recording of action potentials in mammalian neurons using a microbial rhodopsin. Nat. Methods 9, 90-95 (2011)

9. Hochbaum, D. R. et al. All-optical electrophysiology in mammalian neurons using engineered microbial rhodopsins. Nat. Methods 11, 825-833 (2014)

10. Adam, Y. et al. Voltage imaging and optogenetics reveal behaviour-dependent changes in hippocampal dynamics. Nature 569, 413-417 (2019).

11. Zou, P. et al. Bright and fast multicoloured voltage reporters via electrochromic FRET. Nat. Commun. 5, 4625 (2014).
12. Gong, Y. et al. High-speed recording of neural spikes in awake mice and flies with a fluorescent voltage sensor. Science 350, 1361-1366 (2015).

13. Gong, Y., Wagner, M. J., Zhong Li, J. \& Schnitzer, M. J. Imaging neural spiking in brain tissue using FRET-opsin protein voltage sensors. Nat. Commun. 5, 3674 (2014).

14. Abdelfattah, A. S. et al. Bright and photostable chemigenetic indicators for extended in vivo voltage imaging. Science 365, 699-704 (2019).

15. Chow, B. Y. et al. High-performance genetically targetable optical neural silencing by light-driven proton pumps. Nature 463, 98-102 (2010).

16. Platisa, J., Vasan, G., Yang, A. \& Pieribone, V. A. Directed evolution of key residues in fluorescent protein inverses the polarity of voltage sensitivity in the genetically encoded indicator ArcLight. ACS Chem. Neurosci. 8, 513-523 (2017).

17. Wada, T. et al. Crystal structure of the eukaryotic light-driven protonpumping rhodopsin, Acetabularia rhodopsin II, from marine alga. J. Mol. Biol. 411, 986-998 (2011).

18. Grimm, J. B. et al. A general method to improve fluorophores for live-cell and single-molecule microscopy. Nat. Methods 12, 244-250 (2015)

19. Grimm, J. B. et al. A general method to fine-tune fluorophores for live-cell and in vivo imaging. Nat. Methods 14, 987 (2017).

20. Lanyi, J. K. Proton transfers in the bacteriorhodopsin photocycle. Biochim. Biophys. Acta Bioenergetics 1757, 1012-1018 (2006).

21. Piatkevich, K. D. et al. A robotic multidimensional directed evolution approach approach applied to fluorescent voltage reporters. Nat. Chem. Biol. 14, 352-360 (2018)

22. Kannan, M. et al. Fast, in vivo voltage imaging using a red fluorescent indicator. Nat. Methods 15, 1108-1116 (2018).

23. Baker, C. A., Elyada, Y. M., Parra, A. \& Bolton, M. M. L. Cellular resolution circuit mapping with temporal-focused excitation of soma-targeted channelrhodopsin. Elife 5, 1-15 (2016).

24. Lim, S. T., Antonucci, D. E., Scannevin, R. H. \& Trimmer, J. S. A novel targeting signal for proximal clustering of the Kv2.1 K+channel in hippocampal neurons. Neuron 25, 385-397 (2000).

25. Park, J. et al. Screening fluorescent voltage indicators with spontaneously spiking HEK cells. PLoS ONE 8, e85221 (2013).

26. Subach, O. M., Cranfill, P. J., Davidson, M. W. \& Verkhusha, V. V. An enhanced monomeric blue fluorescent protein with the high chemical stability of the chromophore. PLoS ONE 6, e28674 (2011)

27. Wardill, T. J. et al. A neuron-based screening platform for optimizing genetically-encoded calcium indicators. PLOS ONE 8, 1-12 (2013).

28. Hallem, E. A., Ho, M. G. \& Carlson, J. R. The molecular basis of odor coding in the Drosophila antenna. Cell 117, 965-979 (2004).

29. Connelly, T., Savigner, A. \& Ma, M. Spontaneous and sensory-evoked activity in mouse olfactory sensory neurons with defined odorant receptors. $J$. Neurophysiol. 110, 55-62 (2013).

30. Zheng, Q. et al. Rational design of fluorogenic and spontaneously blinking labels for super-resolution imaging. ACS Cent. Sci. 5, 1602-1613 (2019).

\section{Acknowledgements}

We thank the Janelia Cell Culture, Vivarium, Molecular Biology, and Virus Production facilities for assistance. Specifically, we thank Deepika Walpita, Kim Ritola, and Jordan Towne. Funding was provided by the Howard Hughes Medical Institute. We thank Luke Lavis and Brett Mensh for valuable discussions about the manuscript. We thank Luke Lavis, Jonathan Grimm, and Qinsi Zheng for providing Janelia Fluor dyes.

\section{Author contributions}

A.S.A. and E.R.S. designed experiments. A.S.A., R.V., J.Z., A.W., G.P.T., K.P., M.K., and D.S.K. performed and analyzed experiments. A.S.A. and E.R.S. wrote the manuscript.

\section{Competing interests}

A.S.A. and E.R.S. are listed as inventors on a patent application describing positive-going eFRET GEVIs. The other authors declare no competing interests.

\section{Additional information}

Supplementary information is available for this paper at https://doi.org/10.1038/s41467 020-17322-1.

Correspondence and requests for materials should be addressed to A.S.A. or E.R.S.

Peer review information Nature Communications thanks the anonymous reviewer(s) for their contribution to the peer review of this work.

Reprints and permission information is available at http://www.nature.com/reprints

Publisher's note Springer Nature remains neutral with regard to jurisdictional claims in published maps and institutional affiliations. 
(c) (i) Open Access This article is licensed under a Creative Commons Attribution 4.0 International License, which permits use, sharing, adaptation, distribution and reproduction in any medium or format, as long as you give appropriate credit to the original author(s) and the source, provide a link to the Creative Commons license, and indicate if changes were made. The images or other third party material in this article are included in the article's Creative Commons license, unless indicated otherwise in a credit line to the material. If material is not included in the article's Creative Commons license and your intended use is not permitted by statutory regulation or exceeds the permitted use, you will need to obtain permission directly from the copyright holder. To view a copy of this license, visit http://creativecommons.org/ licenses/by/4.0/.

(C) The Author(s) 2020

\section{GENIE Project Team}

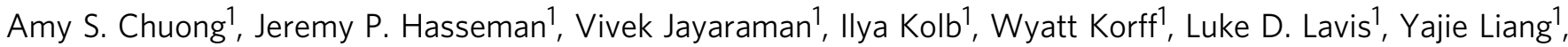
Loren L. Looger', Derek Merryweather', Daniel Reep ${ }^{1}$, Nelson Spruston', Karel Svoboda', Arthur Tsang ${ }^{1}$, Getahun Tsegaye ${ }^{1} \&$ Glenn Turner ${ }^{1}$ 